\title{
Allelopathic potential of mustard crop residues on weed management and performance of transplant Aman rice
}

\author{
M. N. Hossain ${ }^{1}$, M. R. Uddin ${ }^{1}$, M. A. Salam ${ }^{1}$, U. K. Sarker ${ }^{1}$, S. Ferdousi ${ }^{1}$ and M. J. Uddin $^{2}$ \\ ${ }^{1}$ Department of Agronomy, Bangladesh Agricultural University, Mymensingh-2202, Bangladesh \\ ${ }^{2}$ Upazila Agriculture Officer, Ramganj, Laksmipur, Bangladesh
}

\begin{tabular}{|c|c|}
\hline ARTICLE INFO & Abstract \\
\hline $\begin{array}{l}\text { Article history: } \\
\text { Received: } 10 \text { October } 2017 \\
\text { Accepted: } 24 \text { November } 2017\end{array}$ & $\begin{array}{l}\text { Crop allelopathy may be useful to minimize serious problems in the present agricultural production such } \\
\text { as environmental pollution, unsafe production, human health concerns, depletion of crop diversity, soil } \\
\text { sickness and reduction of crop productivity. In this phenomenon an experiment was conducted at the } \\
\text { Agronomy Field Laboratory, Bangladesh Agricultural University, Mymensingh to evaluate the effect of }\end{array}$ \\
\hline $\begin{array}{l}\text { Keywords: } \\
\text { Weed population, Percent } \\
\text { inhibition, Transplanted rice } \\
\text { variety, Yield }\end{array}$ & $\begin{array}{l}\text { consisted of three cultivars of T. aman rice viz., BR11, BR23 and BRRI dhan } 49 \text { and five different level of } \\
\text { mustard crop residues such as no crop residues, mustard crop residues @ } 0.5,1.0,1.5 \text { and } 2.0 \mathrm{t} \mathrm{ha}^{-1} \text {. The } \\
\text { experiment was laid out in a randomized complete block design with three replications. Five weed species } \\
\text { belonging to three families infested the experimental plots. Weed population, weed dry weight and percent } \\
\text { inhibition of weed were significantly influenced by mustard crop residues and cultivar. The maximum }\end{array}$ \\
\hline $\begin{array}{l}\text { Correspondence: } \\
\text { M. R. Uddin } \\
\text { (romijagron@bau.edu.bd) }\end{array}$ & $\begin{array}{l}\text { weed growth was noticed with the cultivar BR23 and the minimum was found in the cultivar BR11. The } \\
\text { grain yield as well as the other yield contributing characters produced by BR11 was the highest among the } \\
\text { studied varieties. The highest percent inhibition of } 71.17,69.19,80.88,70.48 \text { and } 86.97 \text { was in Shama } \\
\text { (Echinochlo acrusgalli), Panishapla (Nymphaea nouchali), Panichaise (Scirpus juncoides), Panikachu } \\
\text { (Monochoria vaginalis) and Susnishak (Marsilea quadrifolia), respectively which was caused by the } \\
\text { application of mustard crop residues @ } 2 \mathrm{t} \mathrm{ha}^{-1} \text {. The highest loss of grain yield was obtained where no } \\
\text { crop residues were incorporated. The highest numbers of tillers hill }^{-1} \text {, numbers of grains panicle }{ }^{-1} \text {, 1000- } \\
\text { grain weight, grain yield, straw yield were observed where mustard crop residues were incorporated @ 2.0 } \\
\mathrm{t} \mathrm{ha}^{-1} \text {. BR11 cultivar with all treatments produced the highest grain and straw yield among the treatment } \\
\text { combination. The results of this study indicate that different amount of mustard crop residues showed }_{\text {potential activity to suppress weed growth. }}\end{array}$ \\
\hline
\end{tabular}

\section{Introduction}

Bangladesh is an agricultural country and its agriculture is predominantly rice based. About $77.07 \%$ of cropped area of Bangladesh is used for rice production. Food security of Bangladesh depends on rice agriculture. Rice (Oryza sativa) is also the staple food for more than two billion people in Asia and four hundred millions of people in Africa and Latin America (IRRI, 2010). The annual production of rice is 34.71 million metric tons from 11.42 million acres of land (BBS, 2015). Transplant aman rice covers 5.53 million acres of land with a production of 13.19 million metric tons (BBS, 2015). In Bangladesh, weed infestation reduces the grain yield by $70-80 \%$ in aus rice, $30-40 \%$ for transplanted aman rice and 22-36\% for modern boro rice (Mamun, 1990; BRRI, 2008).Various factors are responsible for lowering of rice yield and weeds are considered as a major constraint. High competitive ability of weeds exerts a serious negative effect on crop production causing significant losses in crop yield. In present condition herbicide application has become the most widely adopted method for controlling weeds for successful crop production, but their non-judicious use also registers ill effects on soil, water, air, humans and animal health. In view of the numerous problems arising from the chemical weed control, questions have been raised about the continuous use of herbicides. Alternative approaches needs to be considered which is free from such problems. Use of crop allelopathy and allelochemicals for weed control is a sound alternative of chemical herbicide.

Crop residues can interfere with weed development and growth through alteration of soil physical, chemical, and biological characteristics. Crop residues are defined as crop or its parts left in field for decomposition after it has been thrashed or harvested (Kumar and Goh, 2000). Crop residues controls weeds by the release of allelochemicals through decomposition of phytotoxic plant materials (Belz, 2004; Khanh et al., 2005). Currently, researchers are giving more emphasis using different crop residues to suppress weed growth. Information regarding crop residues for suppression of weed is very limited in Bangladesh. However, in Bangladesh, a little attempt has been done to investigate the weed suppressing ability of mustard crop residues and its optimum dose to establish an easy, economic and sustainable method for efficient weed management of $\mathrm{T}$. Aman rice. 


\section{Materials and Methods}

The experiment was conducted at the Agronomy Field Laboratory of Bangladesh Agricultural University, Bangladesh during aman season (June-November) of 2015. The experimental treatment consisted of two factors. In factor A, 5 mustard crop residues- i) No crop residue $\left(\mathrm{T}_{1}\right)$, ii) $0.5 \mathrm{t} \mathrm{ha}^{-1}\left(\mathrm{~T}_{2}\right)$, iii) $1.0 \mathrm{t} \mathrm{ha}^{-1}\left(\mathrm{~T}_{3}\right)$, iv) $1.5 \mathrm{t}$ $\mathrm{ha}^{-1}\left(\mathrm{~T}_{4}\right)$, v) $2.0 \mathrm{t} \mathrm{ha}^{-1}\left(\mathrm{~T}_{5}\right)$ and in factor $\mathrm{B}, 3$ rice variety viz. i) BR11 $\left(\mathrm{V}_{1}\right)$, ii) BR23 $\left(\mathrm{V}_{2}\right)$, iii) BRRI dhan49 $\left(\mathrm{V}_{3}\right)$ were used. The experiment was laid out in a randomized complete block design (RCBD) with three replications. The total number of plot was 45 . Each plot size was $(2.5 \mathrm{~m} \times 2 \mathrm{~m})$. The distance maintained between the individual unit plots was $0.5 \mathrm{~m}$ and distance between the replication was $1.0 \mathrm{~m}$. Land preparation for rice cultivation was done by 3-4 times of ploughing and cross ploughing followed by laddering. The studied crop residues were cut as small as possible by using sickle. The prepared mustard crop residues was applied seven days before transplanting and mixed well to the respective plots by a spade in the prepared field. The experimental plots were fertilized with urea, triple super phosphate, muriate of potash and gypsum @ 150, 52, $82,60 \mathrm{~kg} \mathrm{ha}^{-1}$, respectively for the variety of BR11 and BRRI dhan49. On the other hand $195 \mathrm{~kg}$ urea, $52 \mathrm{~kg}$ triple super phosphate, $82 \mathrm{~kg}$ muriate of potash and 60 $\mathrm{kg}$ gypsum per hectare were applied to the field for BR23. Except urea, the whole amount of other fertilizer was applied before final land preparation. Urea was top dressed in three instalments at 15, 30 and 45 days after transplanting (DAT). Transplanting was done in 30 July 2015 at the rate of three seedlings hill ${ }^{-1}$ maintained row and hill distance of $25 \mathrm{~cm}$ and $15 \mathrm{~cm}$, respectively. Data on weed population, weed dry weight and different parameters of rice plant was taken at different growth stage of rice plants from each plot. After attaining full maturity the crops were harvested on 15 November, 2015. Data on grain and straw yields were collected from an area of $1 \mathrm{~m}^{2}$ in the middle of each plot. The data on other crop characters were randomly sampled from the region outside $1 \mathrm{~m}^{2}$ area (excluding the border hills) which was kept for taking data on grain and straw yields. Data recorded for different parameters were compiled and tabulated in proper form and subjected to statistical analysis. The Analysis of variance was done with the help of computer package MSTAT-C program. The mean differences among the treatments were adjudged by Duncan's Multiple Range Test (DMRT) as laid out by Gomez and Gomez (1984).

\section{Results and Discussion}

\section{Infested weed species in the experimental field}

Five weed species belonging to five families infested the experimental field. Local name, scientific name, family, morphological type and life cycle of the weed in the experimental plot have been presented in Table 1. The weeds of the experimental plots were Echinochloa crusgalli, Nymphaea nouchali, Scirpus juncoides, Monochori vaginalis, Marsilea quadrifolia. Among the weed species 3 were broad leaf, one sedge and one grass type morphology. There were three perennial and two annual weed species in the experimental plot.

\section{Effect of interaction between variety and mustard crop residues on Echinochloa crusgalli}

The interaction between variety and crop residue was found to be significant on weed population, dry weight and percent inhibition. The highest weed population (25.32) was found in $\mathrm{V}_{2} \mathrm{~T}_{1}$ (BR23 $\times$ No crop residues), second highest weed population (21.32) was found in $\mathrm{V}_{1} \mathrm{~T}_{1}$ (BR11 $\times$ No crop residues) treatment (Table 2). The highest weed dry weight (39.32 g) was found in $\mathrm{V}_{2} \mathrm{~T}_{1}$ (BR23 $\times$ No crop residues), and the lowest weed dry weight $(5.68 \mathrm{~g})$ was in $\mathrm{V}_{3} \mathrm{~T}_{5}$ (BRRIdhan $49 \times 2.0 \mathrm{t}$ $\left.\mathrm{ha}^{-1}\right)$. Percent inhibition of weed was the highest in $\mathrm{V}_{2} \mathrm{~T}_{5}$ $\left(\mathrm{BR} 23 \times 2.0\right.$ ton mustardcrop residue $\left.\mathrm{ha}^{-1}\right)$ and the lowest one was observed in $\mathrm{V}_{1} \mathrm{~T}_{1}(\mathrm{BR} 11 \times$ No crop residue) presented in Table 2 . Similar findings were reported by Uddin and Pyon (2010), who found significant weed control efficacy by different crop residues.

Table 1. Infesting weed species found growing in the experimental plots of rice

\begin{tabular}{cccccc}
\hline Sl. No. & Local name & Scientific name & Family & Morphological type & Life cycle \\
\hline 1 & Shama & Echinochloa crusgalli & Poaceae & Grass & Annual \\
2 & Panishapla & Nymphaea nouchali & Nymphaeaceae & Broad leaf & Perennial \\
3 & Chechra & Scirpus juncoides & Cyperaceae & Sedge & Perennial \\
4 & Panikachu & Monochoria vaginalis & Pontederiaceae & Broadleaf & Perennial \\
5 & Susnishak & Marsilea quadrifolia & Marsileaceae & Broadleaf & Annual \\
\hline
\end{tabular}


Table 2. Combined effect of variety and mustard crop residues on Echinochloa crusgalli

\begin{tabular}{|c|c|c|c|}
\hline \multirow{2}{*}{$\begin{array}{c}\text { Treatment } \\
\text { combination }\end{array}$} & \multicolumn{3}{|c|}{ Echinochloa crusgalli } \\
\hline & $\begin{array}{c}\text { Weed population } \\
\left(\text { no. } \mathbf{~ m}^{-2}\right)\end{array}$ & $\begin{array}{l}\text { Dry weight } \\
\left(\mathrm{g} \mathrm{m}^{-2}\right)\end{array}$ & $\%$ inhibition \\
\hline $\mathrm{V}_{1} \mathrm{~T}_{1}$ & $21.32 \mathrm{~b}$ & $33.76 \mathrm{~b}$ & $0.00 \mathrm{k}$ \\
\hline $\mathrm{V}_{1} \mathrm{~T}_{2}$ & $17.32 \mathrm{c}$ & $27.08 \mathrm{c}$ & $18.78 \mathrm{j}$ \\
\hline $\mathrm{V}_{1} \mathrm{~T}_{3}$ & $9.32 \mathrm{e}$ & $14.72 \mathrm{f}$ & $55.73 \mathrm{~g}$ \\
\hline $\mathrm{V}_{1} \mathrm{~T}_{4}$ & $6.68 \mathrm{fg}$ & $10.56 \mathrm{gh}$ & $69.17 \mathrm{~cd}$ \\
\hline $\mathrm{V}_{1} \mathrm{~T}_{5}$ & $4.00 \mathrm{~h}$ & $6.2 \mathrm{ij}$ & $76.53 \mathrm{ab}$ \\
\hline $\mathrm{V}_{2} \mathrm{~T}_{1}$ & $25.32 \mathrm{a}$ & $39.32 \mathrm{a}$ & $0.00 \mathrm{k}$ \\
\hline $\mathrm{V}_{2} \mathrm{~T}_{2}$ & $16.0 \mathrm{c}$ & $24.48 \mathrm{~d}$ & $38.12 \mathrm{i}$ \\
\hline $\mathrm{V}_{2} \mathrm{~T}_{3}$ & $13.32 \mathrm{~d}$ & $20.32 \mathrm{e}$ & $48.46 \mathrm{~h}$ \\
\hline $\mathrm{V}_{2} \mathrm{~T}_{4}$ & $9.32 \mathrm{e}$ & $14.44 \mathrm{f}$ & $62.75 \mathrm{ef}$ \\
\hline $\mathrm{V}_{2} \mathrm{~T}_{5}$ & $5.32 \mathrm{gh}$ & $8.24 \mathrm{hi}$ & $78.75 \mathrm{a}$ \\
\hline $\mathrm{V}_{3} \mathrm{~T}_{1}$ & $21.32 \mathrm{~b}$ & $32.24 \mathrm{~b}$ & $0.00 \mathrm{k}$ \\
\hline $\mathrm{V}_{3} \mathrm{~T}_{2}$ & $16.0 \mathrm{c}$ & $21.84 \mathrm{e}$ & $33.53 \mathrm{i}$ \\
\hline $\mathrm{V}_{3} \mathrm{~T}_{3}$ & $8.0 \mathrm{ef}$ & $10.92 \mathrm{~g}$ & $64.88 \mathrm{de}$ \\
\hline $\mathrm{V}_{3} \mathrm{~T}_{4}$ & $6.68 \mathrm{fg}$ & $9.0 \mathrm{gh}$ & $72.04 \mathrm{bc}$ \\
\hline $\mathrm{V}_{3} \mathrm{~T}_{5}$ & $4.0 \mathrm{~h}$ & $5.68 \mathrm{j}$ & $58.24 \mathrm{fg}$ \\
\hline Level of sig. & $*$ & $* *$ & $* *$ \\
\hline $\mathrm{CV}(\%)$ & 11.18 & 7.44 & 7.76 \\
\hline
\end{tabular}

In a column, figures with the same letter do not differ significantly as per DMRT,

$* *=$ Significant at $1 \%$ level of probability ${ }^{*}=$ Significant at $5 \%$ level of probability, $\mathrm{V}_{1}=\mathrm{BR} 11, \mathrm{~V}_{2}=\mathrm{BR} 23, \mathrm{~V}_{3}=\mathrm{BRRI}$ dhan $49, \mathrm{~T}_{1}=\mathrm{No}$ crop residue, $\mathrm{T}_{2}=0.5 \mathrm{tha}^{-1}, \mathrm{~T}_{3}=1.0 \mathrm{tha}^{-1}, \mathrm{~T}_{4}=1.5 \mathrm{tha}^{-1}, \mathrm{~T}_{5}=2.0 \mathrm{tha}^{-1}$.

Effect of interaction between variety and mustard crop residues on Nymphaea nouchali

The interaction between variety and mustard crop residues was found to be significant on weed population, dry weight and percent inhibition of Nymphaea nouchali. The highest weed population was (25.32) in $\mathrm{V}_{3} \mathrm{~T}_{1}$ (BRRI dhan $49 \times$ no crop residue), second highest weed population (17.31) was found in $\mathrm{V}_{2} \mathrm{~T}_{1}(\mathrm{BR} 23 \times$ no crop residue), and the lowest was found in $\mathrm{V}_{2} \mathrm{~T}_{5}(\mathrm{BR} 23 \times$
$2.0 \mathrm{t} \mathrm{ha}^{-1}$ ) treatment (Table 3). The highest weed dry weight $(6.20 \mathrm{~g})$ was found in $\mathrm{V}_{3} \mathrm{~T}_{1}$ (BRRI dhan $49 \times$ no crop residue), and the lowest weed dry weight was in $\mathrm{V}_{2} \mathrm{~T}_{5}\left(\mathrm{BR} 23 \times 2.0 \mathrm{t} \mathrm{ha}^{-1}\right)$ treatment (Table 3). Percent inhibition of weed was the highest in $\mathrm{V}_{3} \mathrm{~T}_{5}$ (BRRI dhan $49 \times 2.0 \mathrm{t} \mathrm{ha}^{-1}$ ) and the lowest one was observed in $\mathrm{V}_{1} \mathrm{~T}_{1}(\mathrm{BR} 11 \times$ no crop residue) treatment presented in (Table 3).

Table 3. Combined effect of variety and mustard crop residues on Nymphaea nouchali

\begin{tabular}{|c|c|c|c|}
\hline \multirow{2}{*}{$\begin{array}{l}\text { Treatment } \\
\text { combination }\end{array}$} & \multicolumn{3}{|c|}{ Nymphaea nouchali } \\
\hline & $\begin{array}{c}\text { Weed population } \\
\left(\text { no. } \mathbf{~ m}^{-2}\right)\end{array}$ & $\begin{array}{l}\text { Dry weight } \\
\left(\mathrm{g} \mathrm{m}^{-2}\right)\end{array}$ & $\%$ inhibition \\
\hline $\mathrm{V}_{1} \mathrm{~T}_{1}$ & $16.0 \mathrm{bc}$ & $4.20 \mathrm{c}$ & $0.00 \mathrm{~h}$ \\
\hline $\mathrm{V}_{1} \mathrm{~T}_{2}$ & 13.32 cde & $3.2 \mathrm{def}$ & $21.89 \mathrm{~g}$ \\
\hline $\mathrm{V}_{1} \mathrm{~T}_{3}$ & $12.0 \mathrm{def}$ & $2.92 \mathrm{efg}$ & $29.72 \mathrm{f}$ \\
\hline $\mathrm{V}_{1} \mathrm{~T}_{4}$ & $9.32 \mathrm{fg}$ & $2.28 \mathrm{~h}$ & $46.15 \mathrm{~d}$ \\
\hline $\mathrm{V}_{1} \mathrm{~T}_{5}$ & $6.68 \mathrm{gh}$ & $1.60 \mathrm{i}$ & $62.42 \mathrm{~b}$ \\
\hline $\mathrm{V}_{2} \mathrm{~T}_{1}$ & $17.32 \mathrm{~b}$ & $4.80 \mathrm{~b}$ & $0.00 \mathrm{~h}$ \\
\hline $\mathrm{V}_{2} \mathrm{~T}_{2}$ & 13.32 cde & $3.36 \mathrm{de}$ & $28.19 \mathrm{f}$ \\
\hline $\mathrm{V}_{2} \mathrm{~T}_{3}$ & 10.68 ef & $2.56 \mathrm{gh}$ & $44.05 \mathrm{~d}$ \\
\hline $\mathrm{V}_{2} \mathrm{~T}_{4}$ & $6.64 \mathrm{gh}$ & $1.68 \mathrm{i}$ & $65.21 \mathrm{~b}$ \\
\hline $\mathrm{V}_{2} \mathrm{~T}_{5}$ & $5.32 \mathrm{~h}$ & $1.28 \mathrm{i}$ & $72.32 \mathrm{a}$ \\
\hline $\mathrm{V}_{3} \mathrm{~T}_{1}$ & $25.32 \mathrm{a}$ & $6.20 \mathrm{a}$ & $0.00 \mathrm{~h}$ \\
\hline $\mathrm{V}_{3} \mathrm{~T}_{2}$ & $14.68 \mathrm{bcd}$ & $3.64 \mathrm{~d}$ & $39.54 \mathrm{e}$ \\
\hline $\mathrm{V}_{3} \mathrm{~T}_{3}$ & $12.0 \mathrm{def}$ & $2.76 \mathrm{fgh}$ & $51.73 \mathrm{c}$ \\
\hline $\mathrm{V}_{3} \mathrm{~T}_{4}$ & $9.32 \mathrm{fg}$ & $2.22 \mathrm{~h}$ & $62.34 \mathrm{~b}$ \\
\hline $\mathrm{V}_{3} \mathrm{~T}_{5}$ & $6.64 \mathrm{gh}$ & $1.64 \mathrm{i}$ & $72.82 \mathrm{a}$ \\
\hline Level of sig. & $* *$ & $* *$ & $* *$ \\
\hline $\mathrm{CV}(\%)$ & 13.84 & 10.34 & 6.13 \\
\hline
\end{tabular}

In a column, figures with the same letter do not differ significantly as per DMRT,

$* *=$ Significant at $1 \%$ level of probability.

$\mathrm{V}_{1}=\mathrm{BR} 11, \mathrm{~V}_{2}=\mathrm{BR} 23, \mathrm{~V}_{3}=\mathrm{BRRI}$ dhan 49

$\mathrm{T}_{1}=$ No crop residue, $\mathrm{T}_{2}=0.5 \mathrm{tha}^{-1}, \mathrm{~T}_{3}=1.0 \mathrm{tha}^{-1}, \mathrm{~T}_{4}=1.5 \mathrm{tha}^{-1}, \mathrm{~T}_{5}=2.0 \mathrm{tha}^{-1}$. 
Effect of interaction between variety and mustard crop residues on Scirpus juncoides

The interaction between variety and crop residue was found to be significant on weed population, dry weight and percent inhibition. The highest weed population (28.0) was found in $\mathrm{V}_{2} \mathrm{~T}_{1}(\mathrm{BR} 23 \times$ no crop residue) and the lowest was found in $\mathrm{V}_{3} \mathrm{~T}_{5}\left(\mathrm{BRRI}\right.$ dhan $\left.49 \times 2.0 \mathrm{t} \mathrm{ha}^{-1}\right)$ treatment (Table 4$)$. The highest weed dry weight $(8.80$ g) was found in $\mathrm{V}_{2} \mathrm{~T}_{1}$ (BR23 $\times$ no crop residue), which was statistically identical with $\mathrm{V}_{1} \mathrm{~T}_{1}(\mathrm{BR} 11 \times$ no crop residue), and the lowest weed dry weight (1.20 g) was in $\mathrm{V}_{3} \mathrm{~T}_{5}$. Percent inhibition of weed (84.47) was the highest in $\mathrm{V}_{3} \mathrm{~T}_{5}\left(\mathrm{BRRI}\right.$ dhan $\left.49 \times 2.0 \mathrm{t} \mathrm{ha}^{-1}\right)$ and the lowest one was observed in $\mathrm{V}_{1} \mathrm{~T}_{1}(\mathrm{BR} 11 \times$ no crop residue $)$ treatment (Table 4).

Table 4. Combined effect of variety and mustard crop residues on Scirpus juncoides

\begin{tabular}{|c|c|c|c|}
\hline \multirow[b]{2}{*}{ Treatment combination } & \multicolumn{3}{|c|}{ Scirpus juncoides } \\
\hline & $\begin{array}{c}\text { Weed population } \\
\left(\text { no. } \mathbf{m}^{-2}\right)\end{array}$ & $\begin{array}{c}\text { Dry weight } \\
\left(\mathrm{g} \mathrm{m}^{-2}\right)\end{array}$ & \% inhibition \\
\hline $\mathrm{V}_{1} \mathrm{~T}_{1}$ & $24.0 \mathrm{~b}$ & $8.64 \mathrm{a}$ & $0.00 \mathrm{k}$ \\
\hline $\mathrm{V}_{1} \mathrm{~T}_{2}$ & $18.68 \mathrm{~cd}$ & $6.52 \mathrm{c}$ & $23.33 \mathrm{ij}$ \\
\hline $\mathrm{V}_{1} \mathrm{~T}_{3}$ & $16.0 \mathrm{de}$ & $5.40 \mathrm{~d}$ & $36.80 \mathrm{~h}$ \\
\hline $\mathrm{V}_{1} \mathrm{~T}_{4}$ & $10.68 \mathrm{fg}$ & $3.36 \mathrm{fg}$ & $61.10 \mathrm{e}$ \\
\hline $\mathrm{V}_{1} \mathrm{~T}_{5}$ & $5.32 \mathrm{hi}$ & $1.84 \mathrm{hi}$ & $77.06 \mathrm{bc}$ \\
\hline $\mathrm{V}_{2} \mathrm{~T}_{1}$ & $28.0 \mathrm{a}$ & $8.80 \mathrm{a}$ & $0.00 \mathrm{k}$ \\
\hline $\mathrm{V}_{2} \mathrm{~T}_{2}$ & $20.0 \mathrm{c}$ & $7.20 \mathrm{bc}$ & $16.96 \mathrm{j}$ \\
\hline $\mathrm{V}_{2} \mathrm{~T}_{3}$ & $13.32 \mathrm{ef}$ & $4.88 \mathrm{de}$ & $44.20 \mathrm{~g}$ \\
\hline $\mathrm{V}_{2} \mathrm{~T}_{4}$ & $8.0 \mathrm{gh}$ & $2.40 \mathrm{gh}$ & $70.94 \mathrm{~cd}$ \\
\hline $\mathrm{V}_{2} \mathrm{~T}_{5}$ & $5.32 \mathrm{hi}$ & $1.68 \mathrm{hi}$ & $81.12 \mathrm{ab}$ \\
\hline $\mathrm{V}_{3} \mathrm{~T}_{1}$ & $21.32 \mathrm{bc}$ & $7.76 \mathrm{ab}$ & $0.00 \mathrm{k}$ \\
\hline $\mathrm{V}_{3} \mathrm{~T}_{2}$ & $14.68 \mathrm{e}$ & $4.32 \mathrm{ef}$ & $29.16 \mathrm{i}$ \\
\hline $\mathrm{V}_{3} \mathrm{~T}_{3}$ & $10.68 \mathrm{fg}$ & $3.56 \mathrm{f}$ & $52.88 \mathrm{f}$ \\
\hline $\mathrm{V}_{3} \mathrm{~T}_{4}$ & $8.0 \mathrm{gh}$ & $2.32 \mathrm{gh}$ & $69.72 \mathrm{~d}$ \\
\hline $\mathrm{V}_{3} \mathrm{~T}_{5}$ & $4.0 \mathrm{i}$ & $1.20 \mathrm{i}$ & $84.47 \mathrm{a}$ \\
\hline Level of sig. & $* *$ & $*$ & $* *$ \\
\hline $\mathrm{CV}(\%)$ & 12.77 & 13.03 & 9.58 \\
\hline
\end{tabular}

In a column, figures with the same letter do not differ significantly as per DMRT,

$* *=$ Significant at $1 \%$ level of probability; $*=$ Significant at $5 \%$ level of probability

$\mathrm{V}_{1}=\mathrm{BR} 11, \mathrm{~V}_{2}=\mathrm{BR} 23, \mathrm{~V}_{3}=\mathrm{BRRI}$ dhan 49

$\mathrm{T}_{1}=$ No crop residue, $\mathrm{T}_{2}=0.5 \mathrm{tha}^{-1}, \mathrm{~T}_{3}=1.0 \mathrm{tha}^{-1}, \mathrm{~T}_{4}=1.5 \mathrm{tha}^{-1}, \mathrm{~T}_{5}=2.0 \mathrm{tha}^{-1}$.

Effect of interaction between variety and mustard crop residues on Monochoria vaginalis

The interaction between variety and mustard crop residues was found to be significant on weed population, dry weight and percent inhibition of Monochoria vaginalis. The highest weed population (20.0) was found in $\mathrm{V}_{3} \mathrm{~T}_{1}$ (BRRI dhan $49 \times$ no crop residue) and the lowest was found in $\mathrm{V}_{3} \mathrm{~T}_{5}\left(\mathrm{BRRI}\right.$ dhan $\left.49 \times 2.0 \mathrm{t} \mathrm{ha}^{-1}\right)$ treatment (Table 5). The highest weed dry weight $(9.32 \mathrm{~g})$ was found in $\mathrm{V}_{3} \mathrm{~T}_{1}(\mathrm{BRRI}$ dhan $49 \times$ no crop residue) and the lowest dry weight of weed was in $\mathrm{V}_{2} \mathrm{~T}_{5}(\mathrm{BR} 23 \times 2.0 \mathrm{t}$ $\mathrm{ha}^{-1}$ ) treatment (Table 5). Percent inhibition of weed was highest in $\mathrm{V}_{3} \mathrm{~T}_{5}$ and the lowest one was observed in $\mathrm{V}_{2} \mathrm{~T}_{1}$ treatment combination.
Effect of interaction between variety and mustard crop residues on Marsilea quadrifolia

The interactions between variety and crop residue was found to be significant on weed population, dry weight and percent inhibition of Marsilea quadrifolia. The highest weed population (69.32) was found in $V_{3} T_{1}$ (BRRI dhan49 $\times$ no crop residue) followed by $\mathrm{V}_{1} \mathrm{~T}_{1}$ $(\mathrm{BR} 11 \times$ no crop residue) and the lowest was found in $\mathrm{V}_{3} \mathrm{~T}_{5}$ (BRRI dhan49 $\times 2.0 \mathrm{t} \mathrm{ha}^{-1}$ ) treatment (Table 6). The highest weed dry weight $(29.84 \mathrm{~g})$ was found in $\mathrm{V}_{3} \mathrm{~T}_{1}$, and the lowest weed dry weight was in $\mathrm{V}_{3} \mathrm{~T}_{5}$ treatment combination (Table 6). Percent inhibition of weed was the highest in $\mathrm{V}_{3} \mathrm{~T}_{5}\left(\mathrm{BRRI}\right.$ dhan $\left.49 \times 2.0 \mathrm{t} \mathrm{ha}^{-1}\right)$ and the lowest one was observed in $\mathrm{V}_{1} \mathrm{~T}_{1}(\mathrm{BR} 11 \times$ no crop residue) treatment combination. 
Table 5. Combined effect of variety and mustard crop residues on Monochoria vaginalis

\begin{tabular}{|c|c|c|c|}
\hline \multirow{2}{*}{$\begin{array}{c}\text { Treatment } \\
\text { combination }\end{array}$} & \multicolumn{3}{|c|}{ Monochoria vaginalis } \\
\hline & $\begin{array}{c}\text { Weed population } \\
\left(\text { no. } \mathbf{~ m}^{-2}\right)\end{array}$ & $\begin{array}{c}\text { Dry weight } \\
\left(\mathrm{g} \mathrm{m}^{-2}\right)\end{array}$ & $\%$ inhibition \\
\hline $\mathrm{V}_{1} \mathrm{~T}_{1}$ & $17.32 \mathrm{~b}$ & $7.72 \mathrm{~b}$ & $0.00 \mathrm{i}$ \\
\hline $\mathrm{V}_{1} \mathrm{~T}_{2}$ & $16.0 \mathrm{bc}$ & $6.96 \mathrm{bc}$ & $10.28 \mathrm{~h}$ \\
\hline $\mathrm{V}_{1} \mathrm{~T}_{3}$ & $13.20 \mathrm{~d}$ & $5.68 \mathrm{~d}$ & $24.42 \mathrm{~g}$ \\
\hline $\mathrm{V}_{1} \mathrm{~T}_{4}$ & $9.32 \mathrm{e}$ & $3.96 \mathrm{e}$ & $47.48 \mathrm{ef}$ \\
\hline $\mathrm{V}_{1} \mathrm{~T}_{5}$ & $6.68 \mathrm{fg}$ & $2.80 \mathrm{fg}$ & $62.01 \mathrm{~cd}$ \\
\hline $\mathrm{V}_{2} \mathrm{~T}_{1}$ & $16.0 \mathrm{bc}$ & $7.28 \mathrm{bc}$ & $0.00 \mathrm{i}$ \\
\hline $\mathrm{V}_{2} \mathrm{~T}_{2}$ & $9.32 \mathrm{e}$ & $4.28 \mathrm{e}$ & $40.31 \mathrm{f}$ \\
\hline $\mathrm{V}_{2} \mathrm{~T}_{3}$ & 8.0 ef & 3.44 ef & $50.31 \mathrm{e}$ \\
\hline $\mathrm{V}_{2} \mathrm{~T}_{4}$ & $6.68 \mathrm{fg}$ & $2.84 \mathrm{fg}$ & $61.08 \mathrm{~d}$ \\
\hline $\mathrm{V}_{2} \mathrm{~T}_{5}$ & $5.32 \mathrm{gh}$ & $2.24 \mathrm{gh}$ & $69.30 \mathrm{bc}$ \\
\hline $\mathrm{V}_{3} \mathrm{~T}_{1}$ & $20.0 \mathrm{a}$ & $9.32 \mathrm{a}$ & $0.00 \mathrm{i}$ \\
\hline $\mathrm{V}_{3} \mathrm{~T}_{2}$ & $14.68 \mathrm{~cd}$ & $6.84 \mathrm{c}$ & $23.78 \mathrm{~g}$ \\
\hline $\mathrm{V}_{3} \mathrm{~T}_{3}$ & 8.0 ef & 3.48 ef & $64.30 \mathrm{~cd}$ \\
\hline $\mathrm{V}_{3} \mathrm{~T}_{4}$ & $5.32 \mathrm{gh}$ & $2.32 \mathrm{gh}$ & $74.64 \mathrm{ab}$ \\
\hline $\mathrm{V}_{3} \mathrm{~T}_{5}$ & $4.0 \mathrm{~h}$ & $1.80 \mathrm{~h}$ & $80.12 \mathrm{a}$ \\
\hline Level of sig. & $* *$ & ** & ** \\
\hline $\mathrm{CV}(\%)$ & 12.25 & 9.92 & 10.75 \\
\hline
\end{tabular}

In a column, figures with the same letter do not differ significantly as per DMRT,

$* *=$ Significant at $1 \%$ level of probability.

$\mathrm{V}_{1}=\mathrm{BR} 11, \mathrm{~V}_{2}=\mathrm{BR} 23, \mathrm{~V}_{3}=$ BRRI dhan 49

$\mathrm{T}_{1}=$ No crop residue, $\mathrm{T}_{2}=0.5 \mathrm{tha}^{-1}, \mathrm{~T}_{3}=1.0 \mathrm{tha}^{-1}, \mathrm{~T}_{4}=1.5 \mathrm{tha}^{-1}, \mathrm{~T}_{5}=2.0 \mathrm{tha}^{-1}$.

Table 6. Combined effect of variety and mustard crop residues on Marsilea quadrifolia

\begin{tabular}{|c|c|c|c|}
\hline \multirow{2}{*}{$\begin{array}{c}\text { Treatment } \\
\text { combination }\end{array}$} & \multicolumn{3}{|c|}{ Marsilea quadrifolia } \\
\hline & $\begin{array}{l}\text { Weed population } \\
\left(\text { no } \mathbf{~ m}^{-2}\right)\end{array}$ & $\begin{array}{c}\text { Dry weight } \\
\left(\mathrm{g} \mathrm{m}^{-2}\right)\end{array}$ & $\%$ inhibition \\
\hline $\mathrm{V}_{1} \mathrm{~T}_{1}$ & $58.68 \mathrm{~b}$ & $24.0 \mathrm{~b}$ & $0.00 \mathrm{j}$ \\
\hline$V_{1} T_{2}$ & $30.68 \mathrm{~d}$ & $13.24 \mathrm{de}$ & $29.33 \mathrm{i}$ \\
\hline $\mathrm{V}_{1} \mathrm{~T}_{3}$ & $25.32 \mathrm{~d}$ & $10.48 \mathrm{e}$ & $56.30 \mathrm{e}$ \\
\hline $\mathrm{V}_{1} \mathrm{~T}_{4}$ & $14.68 \mathrm{e}$ & $6.04 \mathrm{fg}$ & $74.84 \mathrm{c}$ \\
\hline $\mathrm{V}_{1} \mathrm{~T}_{5}$ & $9.32 \mathrm{efg}$ & $3.68 \mathrm{gh}$ & $83.98 \mathrm{~b}$ \\
\hline $\mathrm{V}_{2} \mathrm{~T}_{1}$ & $54.68 \mathrm{~b}$ & $23.36 \mathrm{~b}$ & $0.00 \mathrm{j}$ \\
\hline$V_{2} T_{2}$ & $37.32 \mathrm{c}$ & $15.32 \mathrm{~cd}$ & $32.79 \mathrm{~h}$ \\
\hline$V_{2} T_{3}$ & $28.0 \mathrm{~d}$ & $10.80 \mathrm{e}$ & $52.68 \mathrm{f}$ \\
\hline $\mathrm{V}_{2} \mathrm{~T}_{4}$ & $14.68 \mathrm{e}$ & $6.52 \mathrm{f}$ & $72.09 \mathrm{c}$ \\
\hline $\mathrm{V}_{2} \mathrm{~T}_{5}$ & $8.0 \mathrm{fg}$ & $3.44 \mathrm{gh}$ & $85.21 \mathrm{~b}$ \\
\hline $\mathrm{V}_{3} \mathrm{~T}_{1}$ & $69.32 \mathrm{a}$ & $29.84 \mathrm{a}$ & $0.00 \mathrm{j}$ \\
\hline $\mathrm{V}_{3} \mathrm{~T}_{2}$ & $42.68 \mathrm{c}$ & $17.16 \mathrm{c}$ & $41.37 \mathrm{~g}$ \\
\hline $\mathrm{V}_{3} \mathrm{~T}_{3}$ & $28.0 \mathrm{~d}$ & $11.64 \mathrm{e}$ & $60.42 \mathrm{~d}$ \\
\hline $\mathrm{V}_{3} \mathrm{~T}_{4}$ & $13.32 \mathrm{ef}$ & $5.24 \mathrm{fgh}$ & $82.35 \mathrm{~b}$ \\
\hline $\mathrm{V}_{3} \mathrm{~T}_{5}$ & $6.68 \mathrm{~g}$ & $2.56 \mathrm{~h}$ & $91.73 \mathrm{a}$ \\
\hline Level of sig. & $* *$ & $* *$ & ** \\
\hline $\mathrm{CV}(\%)$ & 12.17 & 12.74 & 3.66 \\
\hline
\end{tabular}

In a column, figures with the same letter do not differ significantly as per DMRT

$* *=$ Significant at $1 \%$ level of probability.

$\mathrm{V}_{1}=\mathrm{BR} 11, \mathrm{~V}_{2}=\mathrm{BR} 23, \mathrm{~V}_{3}=$ BRRI dhan 49

$\mathrm{T}_{1}=$ No crop residue, $\mathrm{T}_{2}=0.5 \mathrm{tha}^{-1}, \mathrm{~T}_{3}=1.0 \mathrm{tha}^{-1}, \mathrm{~T}_{4}=1.5 \mathrm{tha}^{-1}, \mathrm{~T}_{5}=2.0 \mathrm{tha}^{-1}$.

Yield and Yield Contributing Characters at Harvest

Grain yield as influenced by variety

The studied variety differed significantly in respect of grain yield. The highest grain yield $\left(4.09 \mathrm{t} \mathrm{ha}^{-1}\right)$ was obtained in BR11 (Fig. 1). The increased yield might be due to the lowest number of sterile spikelet panicle ${ }^{-1}$. The lowest grain yield $\left(3.72 \mathrm{t} \mathrm{ha}^{-1}\right)$ was obtained in BRRI dhan49. This difference was observed due to different varietal characteristics of rice plant. BRRI (2005) also reported variation in grain yield among the varieties. 
Grain yield as influenced by mustard crop residues

Grain yield was significantly influenced by mustard crop residues. The highest grain yield $\left(4.14 \mathrm{t} \mathrm{ha}^{-1}\right)$ was produced by $\mathrm{T}_{5}\left(2.0 \mathrm{t} \mathrm{ha}^{-1}\right)$ treatment and the lowest grain yield $\left(3.52 \mathrm{t} \mathrm{ha}^{-1}\right.$ ) was produced by $\mathrm{T}_{1}$ (no crop residue) treatment (Fig 2). Incorporation of 2.0 ton mustard crop residue $\mathrm{ha}^{-1}$ decrease weed emergence in the rice field and produced maximum grain yield also. It might be due to application of crop residues added organic matter to the soil and enhance grain yield. On the other hand, control plot (no crop residue) showed maximum weed population and highest dry weight of weed. The weeds compete with the crop for nutrient, water, air, sunlight and space and so grain yield decreased. Uddin and Pyon (2010) also reported the similar results, where crop residues influenced in crop performance.

\section{Straw yield as influenced by mustard crop residues} Straw yield was significantly influenced by mustard crop residues (Fig. 3). The highest straw yield (7.51 t ha ${ }^{-1}$ ) was observed in 2.0 ton mustard crop residue ha ${ }^{-1}$ treatment and the lowest straw yield $\left(6.41 \mathrm{t} \mathrm{ha}^{-1}\right)$ was observed in $\mathrm{T}_{1}$ (no crop residue) treatment.

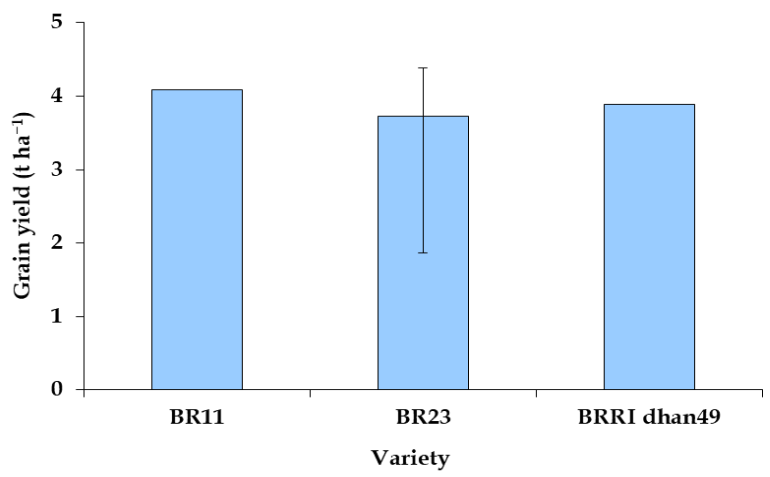

Fig. 1. Grain yield as influenced by variety (Bar represents standard error mean)

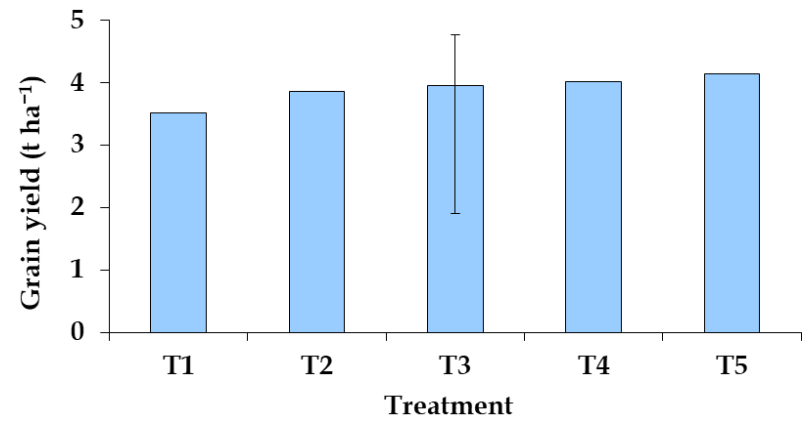

Fig. 2. Grain yield as influenced by mustard crop residues (Bar represents Standard error mean)

Here, $\mathrm{T}_{1}=$ No crop residue, $\mathrm{T}_{2}=0.5 \mathrm{t} \mathrm{ha}^{-1}, \mathrm{~T}_{3}=1.0 \mathrm{t} \mathrm{ha}^{-1}$, $\mathrm{T}_{4}=1.5 \mathrm{tha}^{-1}, \mathrm{~T}_{5}=2.0 \mathrm{tha}^{-1}$.

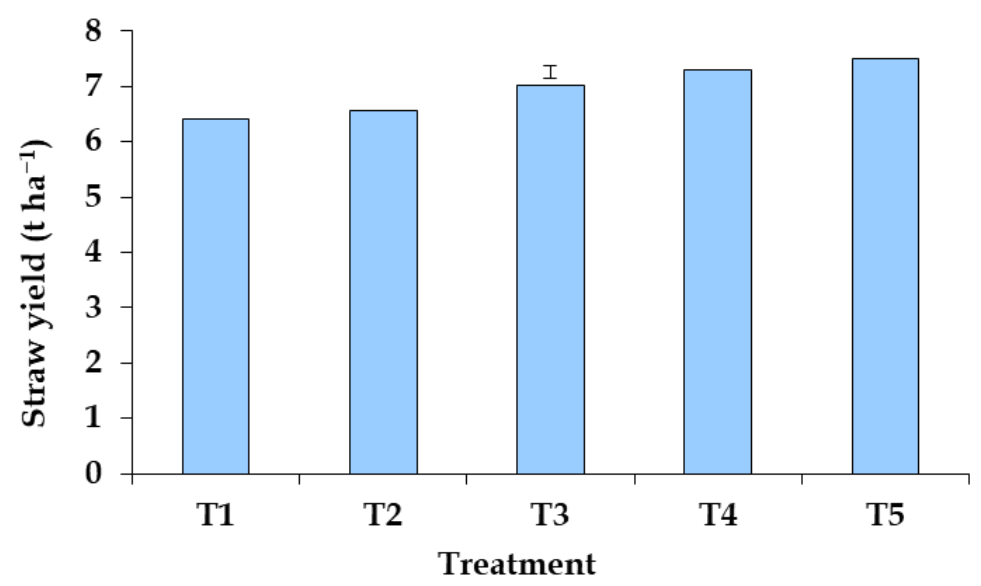

Fig. 3. Straw yield as influenced by mustard crop residues (Bar represents standard error mean) Here, $\mathrm{T}_{1}=$ No crop residue, $\mathrm{T}_{2}=0.5 \mathrm{tha}^{-1}, \mathrm{~T}_{3}=1.0 \mathrm{t} \mathrm{ha}^{-1}, \mathrm{~T}_{4}=1.5 \mathrm{tha}^{-1}, \mathrm{~T}_{5}=2.0 \mathrm{t} \mathrm{ha}^{-1}$. 
Table 7. Combined effect of variety and mustard crop residues on yield and yield contributing characters of T. aman rice

\begin{tabular}{|c|c|c|c|c|c|c|c|c|c|}
\hline $\begin{array}{c}\text { Treatment } \\
\text { combination }\end{array}$ & $\begin{array}{c}\text { Plant } \\
\text { height } \\
(\mathrm{cm})\end{array}$ & $\begin{array}{c}\text { Effective } \\
\text { tillers hill' }^{-1} \\
\text { (no.) }\end{array}$ & $\begin{array}{c}\text { Panicle } \\
\text { length } \\
(\mathrm{cm}) \\
\end{array}$ & $\begin{array}{c}\text { Grains } \\
\text { panicle }^{-1} \\
\text { (no.) }\end{array}$ & $\begin{array}{l}\text { 1000-grain } \\
\text { weight (g) }\end{array}$ & $\begin{array}{c}\text { Grain } \\
\text { yield } \\
\left(\mathrm{t} \mathrm{ha}^{-1}\right)\end{array}$ & $\begin{array}{c}\text { Straw } \\
\text { yield } \\
\left(\mathrm{t} \mathrm{ha}^{-1}\right)\end{array}$ & $\begin{array}{c}\text { Biological } \\
\text { yield } \\
\left(\mathrm{t} \mathrm{ha}^{-1}\right) \\
\end{array}$ & $\begin{array}{c}\text { Harvest } \\
\text { index }(\%)\end{array}$ \\
\hline $\mathrm{V}_{1} \mathrm{~T}_{1}$ & 100.00 & 8.09 & 22.13 & $99.46 \mathrm{gh}$ & 24.45 & $3.65 \mathrm{~g}$ & $6.44 \mathrm{gh}$ & $10.10 \mathrm{gh}$ & $36.19 b c$ \\
\hline $\mathrm{V}_{1} \mathrm{~T}_{2}$ & 100.90 & 8.14 & 22.18 & 108.90 ef & 24.55 & $4.00 \mathrm{~cd}$ & $6.60 \mathrm{efg}$ & 10.60 ef & $37.74 \mathrm{a}$ \\
\hline $\mathrm{V}_{1} \mathrm{~T}_{3}$ & 101.70 & 8.22 & 22.31 & $112.10 \mathrm{e}$ & 24.68 & $4.17 \mathrm{~b}$ & $7.42 \mathrm{bc}$ & $11.59 \mathrm{bc}$ & $36.01 \mathrm{bc}$ \\
\hline $\mathrm{V}_{1} \mathrm{~T}_{4}$ & 102.40 & 8.35 & 22.50 & $114.90 \mathrm{de}$ & 24.77 & $4.24 \mathrm{ab}$ & $7.71 \mathrm{ab}$ & $11.95 \mathrm{ab}$ & $35.51 \mathrm{c}$ \\
\hline $\mathrm{V}_{1} \mathrm{~T}_{5}$ & 103.40 & 8.60 & 22.72 & $117.00 \mathrm{de}$ & 24.83 & $4.38 \mathrm{a}$ & $7.97 \mathrm{a}$ & $12.34 \mathrm{a}$ & $35.47 \mathrm{c}$ \\
\hline $\mathrm{V}_{2} \mathrm{~T}_{1}$ & 96.07 & 6.59 & 22.26 & $90.81 \mathrm{i}$ & 22.22 & $3.48 \mathrm{~h}$ & $6.16 \mathrm{~h}$ & $9.64 \mathrm{~h}$ & $36.12 b c$ \\
\hline $\mathrm{V}_{2} \mathrm{~T}_{2}$ & 96.67 & 6.73 & 22.34 & 94.12 hi & 22.43 & $3.69 \mathrm{~g}$ & $6.25 \mathrm{gh}$ & $9.94 \mathrm{gh}$ & $37.11 \mathrm{ab}$ \\
\hline $\mathrm{V}_{2} \mathrm{~T}_{3}$ & 97.25 & 6.86 & 22.45 & 96.92 ghi & 22.51 & $3.74 \mathrm{fg}$ & $6.53 \mathrm{fgh}$ & $10.27 \mathrm{fg}$ & $36.38 \mathrm{bc}$ \\
\hline $\mathrm{V}_{2} \mathrm{~T}_{4}$ & 97.73 & 7.00 & 22.53 & $99.98 \mathrm{gh}$ & 22.64 & $3.80 \mathrm{efg}$ & $6.98 \mathrm{de}$ & $10.79 \mathrm{de}$ & $35.26 \mathrm{c}$ \\
\hline $\mathrm{V}_{2} \mathrm{~T}_{5}$ & 98.27 & 7.12 & 22.58 & $102.60 \mathrm{fg}$ & 22.73 & $3.91 \mathrm{de}$ & $7.10 \mathrm{~cd}$ & $11.01 \mathrm{de}$ & $35.48 \mathrm{c}$ \\
\hline $\mathrm{V}_{3} \mathrm{~T}_{1}$ & 97.87 & 8.73 & 22.14 & $121.40 \mathrm{~d}$ & 24.12 & $3.41 \mathrm{~h}$ & $6.62 \mathrm{efg}$ & $10.03 \mathrm{gh}$ & $34.00 \mathrm{~d}$ \\
\hline$V_{3} T_{2}$ & 98.30 & 8.95 & 22.21 & $136.50 \mathrm{c}$ & 24.23 & $3.89 \mathrm{def}$ & $6.84 \mathrm{def}$ & $10.74 \mathrm{def}$ & $36.23 \mathrm{bc}$ \\
\hline$V_{3} T_{3}$ & 99.97 & 9.09 & 22.34 & $143.30 \mathrm{bc}$ & 24.33 & $3.96 \mathrm{de}$ & $7.11 \mathrm{~cd}$ & $11.07 \mathrm{de}$ & $35.79 \mathrm{bc}$ \\
\hline $\mathrm{V}_{3} \mathrm{~T}_{4}$ & 101.40 & 9.21 & 22.40 & $149.40 \mathrm{ab}$ & 24.41 & $4.00 \mathrm{~cd}$ & $7.21 \mathrm{~cd}$ & $11.21 \mathrm{~cd}$ & $35.70 \mathrm{c}$ \\
\hline $\mathrm{V}_{3} \mathrm{~T}_{5}$ & 102.00 & 9.70 & 22.56 & $153.40 \mathrm{a}$ & 24.53 & $4.15 \mathrm{bc}$ & $7.47 \mathrm{bc}$ & $11.61 \mathrm{bc}$ & $35.70 \mathrm{c}$ \\
\hline Level of sig. & NS & NS & NS & $*$ & NS & $*$ & * & $*$ & $*$ \\
\hline CV $(\%)$ & 2.29 & 3.37 & 1.45 & 3.84 & 1.31 & 2.25 & 3.13 & 2.49 & 1.96 \\
\hline
\end{tabular}

In a column, figures with same letter(s) or without letter do not differ significantly where figures with dissimilar letter differ significantly as per DMRT

$*$ = Significant at $5 \%$ level of probability, NS = not significant.

Here, $\mathrm{V}_{1}=\mathrm{BR} 11, \mathrm{~V}_{2}=\mathrm{BR} 23, \mathrm{~V}_{3}=\mathrm{BRRI}$ dhan 49

$\mathrm{T}_{1}=$ No crop residue, $\mathrm{T}_{2}=0.5 \mathrm{tha}^{-1}, \mathrm{~T}_{3}=1.0 \mathrm{tha}^{-1}, \mathrm{~T}_{4}=1.5 \mathrm{tha}^{-1}, \mathrm{~T}_{5}=2.0 \mathrm{tha}^{-1}$.

\section{Conclusion}

Weed population, weed dry weight, percent inhibition were significantly affected by variety, mustard crop residues and their interaction. It is found from this study that incorporation of mustard crop residue was really able to suppress weed infestation. Mustard crop residues might be a successful weed management tool which can inhibit the germination of several weed species and not inhibit the growth performance of the crop. It may be useful to minimize hazardous environmental issues, excessive uses of synthetic herbicide and provide better soil health. So it is clear from the study that mustard crop residues have an immense prospect on weed management and sustainable agricultural development. In addition, further research using different crop residues needs to be done for getting conclusive result.

\section{References}

BBS (Bangladesh Bureau of Statistics). 2015. Statistical Year Book of Bangladesh, Bur. Stat., Stat. Div., Min. Plan., Govt. People's Repub., Bangladesh, Dhaka. pp: 37.

Belz, RG. 2004. Evaluation of allelopathic traits in Triticum L. spp and Secale cereal L. PhD Thesis, University of Hohenheim, Stuttgart, Germany.

BRRI (Bangladesh Rice Research Institute) 2005. Adhunik Dhaner Chash. Bangladesh Rice Res. Inst., Joydevpur, Gazipur, Bangladesh. Pub. No. 10.pp: 12, 20-21, 23.
BRRI (Bangladesh Rice Research Institute) 2008. Annual Report for 2007.Bangladesh Rice Res. Inst., Joydevpur, Bangladesh. pp: $28-35$.

Gomez, K.A. and Gomez, A.A. 1984. Duncan's, Muitiple Range Test. Statistical Procedures for Agril.Res. $2^{\text {nd }} E d n$.A Wiley InterScience publication. John Wiley and Sons. New York. pp: 202-215.

IRRI (International Rice Research Institute) 2010. Rice Yield by country and geographical region. World Rice Statistic. Intl. Rice Res. Inst., Los Banos, Laguna Philippines. pp: 1-8.

Khanh, T.D., Chung, M.I., Xuan, T.D. and Tawata, S. 2005. The exploitation of crop allelopathy in sustainable agricultural production. J.Agron.Crop Sci. 191: 172-184.

Kumar, K. and Goh, K.M. 2000. Crop residues and management practices : effects on soil quality, soil nitrogen dynamics, crop yield, and nitrogen recovery. Adv. Agron. 68: 197319.

Mamun, A.A. 1990. Weeds and their control: A review of weed research in Bangladesh. Agricultural and Rural Development in Bangladesh. Japan Intl. Cooperation Agency, Dhaka, Bangladesh. JSARD. 19: 45-72.

Uddin, M.R. and Pyon, J.Y. 2010. Herbicidal activity of rotation crop residues on weeds and selectivity to crops. J. Agril. Sci. 37(1): $1-6$. 